\title{
BISTATIC SYNTHETIC APERTURE HITCHHIKER IMAGING
}

\author{
Can Evren Yarman ${ }^{1}$, Birsen Yazıcı ${ }^{1}$, Margaret Cheney ${ }^{2}$ \\ Rensselaer Polytechnic Institute, Troy, NY. \\ ${ }^{1}$ Electrical, Computer and System Engineering Department, \\ ${ }^{2}$ Department of Mathematical Sciences
}

\begin{abstract}
We introduce a new bistatic synthetic-aperture imaging method for a radar system consisting of two receivers, which will be referred to as hitchhikers, and a source of opportunity. We assume the receivers fly along arbitrary, but known, flight trajectories.

We develop a correlation-based filtered-backprojection reconstruction method that preserves the visible edges of the target scene in the reconstructed image. We present an analysis of the computational complexity of the introduced method and demonstrate its applicability in numerical simulations.

Potential applications of the proposed method include image formation using low earth orbiting and space-borne satellites as sources of opportunity.
\end{abstract}

Index Terms - Bistatic synthetic aperture radar, correlation filtering, tomography, filtered backprojection, hitchhiking.

\section{INTRODUCTION}

A radar whose transmitter and receiver are sufficiently separated is called a bistatic radar. When a bistatic radar is mounted on space or airborne platform(s) it is called a bistatic synthetic-aperture radar (BISAR). A hitchhiker is a receiver that uses sources (/transmitters) of opportunity to detect and locate targets $[15,16,11,6]$. For the rest of the presentation we will use the terms "source" and "transmitter" interchangeably.

In this paper, we consider a synthetic-aperture imaging system consisting of two receivers traversing arbitrary flight trajectories that use a source of opportunity for imaging as illustrated in Figure 1. Due to its combined BISAR and hitchhiking structure, we refer to the system under consideration as bistatic synthetic aperture hitchhiker (BISAH).

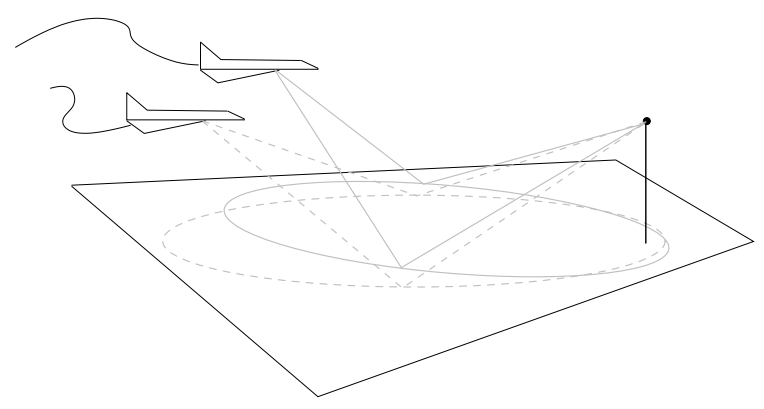

Fig. 1. Bistatic synthetic aperture hitchhiker geometry.

This work is supported by Air Force Office of Scientific Research under the agreements F49620-03-1-0051 and FA9550-06-1-0017.
While, for the current discussion, we consider a static cooperative transmitter, where the information (location of the transmitter, transmitted waveforms, antenna beam pattern, etc.) about the source is available, the method we introduce is also applicable to multiple static and/or mobile cooperative and/or non-cooperative transmitters (such as transmitters on low-earth-orbiting and space-borne satellites $[4,5])$, where information about the sources is not available. We present a filtered-backprojection (FBP) reconstruction method that preserves the visible edges of the target scene at the correct location and orientation in the reconstructed image.

Our work provides a tomographic approach to correlation-based imaging methods and introduces a new FBP-type reconstruction method. In particular, we have combined the "correlation" methods presented in $[1,3]$ with microlocal techniques $[8,9]$ to develop FBPtype reconstruction methods for BISAH. Given a pair of receivers, the spatial-correlation method compares the received signals to identify a target within the illuminated scene, eliminating the need for knowledge about the transmitter location and waveform. Microlocal techniques provide an approximate FBP-type inversion method; however, if an exact inversion is possible, the result often reduces to the exact inversion formula. Furthermore, the FBP-type inversions have the desirable property that visible edges in the target scene not only appear at the right location and at the right orientation but also at the right strength in the reconstructed image. Thus, we perform reconstruction in three steps: First correlate the received signals; next filter the correlated signal; and finally backproject the correlated and filtered signals along the intersection of the illuminated surface and the hyperboloid $H_{12}$ defined in Section 3. We compare our method to the BISAR reconstruction method introduced in [17], present the analysis of computational complexity for both methods, and compare their performances in numerical simulations.

The organization of the paper is as follows. In Section 2, we start with the introduction of the system under consideration and present the forward model. In Section 3, we present the correlation-FBPtype image reconstruction methods for cooperative sources of opportunity. In Section 4, we compare our method with the matched-filterFBP reconstruction method for bistatic synthetic-aperture radar. In Section 5, we demonstrate the performance of our method in numerical simulations. Finally, we conclude our discussion in Section 6.

\section{FORWARD MODEL}

Let $\gamma_{R_{1}}(s)$ and $\gamma_{R_{2}}(s), s \in \mathbb{R}$ be the BISAH trajectories. Let $\mathbf{x}=$ $(\boldsymbol{x}, \psi(\boldsymbol{x})) \in \mathbb{R}^{3}$ denote the surface of the earth, where $\boldsymbol{x}=\left(x_{1}, x_{2}\right)$ and $\psi: \mathbb{R}^{2} \rightarrow \mathbb{R}$ is a known smooth function.

We assume that the electromagnetic waves propagate in free space and then scatter in a thin region at the earth's surface. Under the startstop approximation, the single-scattering (Born) approximation of 
the received signal at the $i^{\text {th }}(i=1,2)$ receiver due to a transmitter located at $\mathbf{y} \in \mathbb{R}^{3}$ can be modeled as [8]:

$$
d_{i}(s, t) \approx \int \mathrm{e}^{-\mathrm{i} 2 \pi \omega\left(t-r_{i, \mathbf{y}}(s, \boldsymbol{x}) / c_{0}\right)} A_{i, \mathbf{y}}(\omega, s, \boldsymbol{x}) G(\boldsymbol{x}) d \omega d \boldsymbol{x},
$$

where $\mathrm{i}=\sqrt{-1}, t$ is the fast-time variable, $s$ is the slow-time variable that parameterizes the trajectory, $r_{i, \mathbf{y}}(s, \mathbf{x})=|\mathbf{y}-\mathbf{x}|+\mid \mathbf{x}-$ $\gamma_{R_{i}}(s) \mid$ is the total travel time, $c_{0}$ denotes the speed of light, $G(\boldsymbol{x})$ denotes the ground reflectivity, and

$$
A_{i}(\omega, s, \boldsymbol{x})=\frac{\pi}{2} \omega^{2} \frac{J_{i}(\omega, s, \boldsymbol{x})}{\left|\gamma_{R_{i}}(s)-\mathbf{x}\right|} \frac{J_{\mathbf{y}}(\omega, s, \boldsymbol{x})}{|\mathbf{x}-\mathbf{y}|},
$$

where $J_{i}$ is the receiver antenna beam pattern and $J_{\mathbf{y}}$ is the transmitter antenna beam pattern (which also includes the transmitter waveform).

The ideal image reconstruction problem is to estimate $G$ from the knowledge of $d_{i}(s, t), i=1,2$, for some range $\left[s_{a}, s_{b}\right]$ and $\left[0, t_{0}\right]$ of $s$ and $t$, respectively. For monostatic SAR and BISAR, the general strategy for estimating $G$ is to perform matched filtering followed by FBP (MF-FBP) $[12,8,17]$. In this paper we will take an alternative approach to MF-FBP: instead, we form our images by the method described above, namely correlation followed by FBP. We refer to the resulting method as C-FBP. We discuss this in more detail below.

\section{IMAGE FORMATION VIA C-FBP}

Define the correlation of $d_{1}$ and $d_{2}$ by

$$
d_{12}\left(s, s^{\prime}, t\right)=\int \underline{d}_{1}(s, \tau) \underline{d}_{2}^{*}\left(s+s^{\prime}, \tau-t\right) d \tau,
$$

where ${ }^{*}$ denotes complex conjugation, and $\underline{d}_{i}(s, t)$ is

$$
\underline{d}_{i}(s, t)=d_{i}(s, t)-E\left[d_{i}(s, t)\right], \quad i=1,2 .
$$

Let $C_{G}$ denote the auto-covariance of $G$, i.e.

$$
C_{G}\left(\boldsymbol{x}, \boldsymbol{x}^{\prime}\right)=E\left[(G(\boldsymbol{x})-E[G(\boldsymbol{x})])\left(G\left(\boldsymbol{x}^{\prime}\right)-E\left[G\left(\boldsymbol{x}^{\prime}\right)\right]\right)^{*}\right] .
$$

Then

$$
\begin{aligned}
E\left[d_{12}\left(s, s^{\prime}, t\right)\right]=\int & \mathrm{e}^{-\mathrm{i} 2 \pi \omega\left(t-\left[\left|\mathbf{x}-\gamma_{R_{1}}(s)\right|-\left|\mathbf{x}^{\prime}-\gamma_{R_{2}}\left(s+s^{\prime}\right)\right|\right] / c_{0}\right)} \\
& \times A_{12}\left(\omega, s, s^{\prime}, \boldsymbol{x}, \boldsymbol{x}^{\prime}\right) C_{G}\left(\boldsymbol{x}, \boldsymbol{x}^{\prime}\right) d \omega d \boldsymbol{x} d \boldsymbol{x}^{\prime},
\end{aligned}
$$

where

$$
\begin{aligned}
A_{12}\left(\omega, s, s^{\prime}, \boldsymbol{x}, \boldsymbol{x}^{\prime}\right)=\mathrm{e}^{\mathrm{i} 2 \pi \omega\left(\left[|\mathbf{y}-\mathbf{x}|-\left|\mathbf{y}-\mathbf{x}^{\prime}\right|\right] / c_{0}\right)} & \\
& \times A_{1}(\omega, s, \boldsymbol{x}) A_{2}^{*}\left(\omega, s+s^{\prime}, \boldsymbol{x}^{\prime}\right)
\end{aligned}
$$

We make the incoherent-field approximation [2] to (6) by assuming that $G$ is statistically uncorrelated in $\boldsymbol{x}$ :

$$
E\left[G(\boldsymbol{x}) G\left(\boldsymbol{x}^{\prime}\right)^{*}\right]=E[G(\boldsymbol{x})] E\left[G\left(\boldsymbol{x}^{\prime}\right)\right]^{*} .
$$

Thus we write $C_{G}\left(\boldsymbol{x}, \boldsymbol{x}^{\prime}\right)=R_{G}(\boldsymbol{x}) \delta\left(\boldsymbol{x}-\boldsymbol{x}^{\prime}\right)$ and simplify (6) to

$$
\begin{aligned}
\mathcal{F}\left[R_{G}\right]\left(s, s^{\prime}, t\right)=\int & \mathrm{e}^{-\mathrm{i} 2 \pi \omega\left(t-r_{12}\left(s, s^{\prime}, \boldsymbol{x}\right) / c_{0}\right)} \\
& \times A_{12}\left(\omega, s, s^{\prime}, \boldsymbol{x}, \boldsymbol{x}\right) R_{G}(\boldsymbol{x}) d \omega d \boldsymbol{x},
\end{aligned}
$$

where $r_{12}\left(s, s^{\prime}, \boldsymbol{x}\right)=\left|\mathbf{x}-\gamma_{R_{1}}(s)\right|-\left|\mathbf{x}-\gamma_{R_{2}}\left(s+s^{\prime}\right)\right|$ and $R_{G}(\boldsymbol{x})=E\left[|G(\boldsymbol{x})-E[G(\boldsymbol{x})]|^{2}\right]$ is referred to as the radiant exitance or radiance of the object [2].

For some $m_{A}$, we assume that $A_{12}$ satisfies

$$
\begin{array}{r}
\sup _{(s, \boldsymbol{x}) \in K}\left|\partial_{\omega}^{\alpha} \partial_{s}^{\beta} \partial_{s^{\prime}}^{\beta^{\prime}} \partial_{x_{1}}^{\rho_{1}} \partial_{x_{2}}^{\rho_{2}} A_{12}\left(\omega, s, s^{\prime}, \boldsymbol{x}, \boldsymbol{x}\right)\right| \\
\leq C_{A}\left(1+\omega^{2}\right)^{\left(m_{A}-|\alpha|\right) / 2}
\end{array}
$$

where $K$ is any compact subset of $\mathbb{R} \times \mathbb{R}^{2}$, and the constant $C_{A}$ depends on $K, \alpha, \beta, \beta^{\prime}, \rho_{1}$, and $\rho_{2}$. This assumption is needed in order to make various stationary phase calculations hold. In practice (10) is satisfied for transmitters and receivers sufficiently far from the ground, especially for air- or space-borne transmitters and receivers.

Equation (9) defines $\mathcal{F}$ as a Fourier integral operator [13] whose leading-order contribution comes from those points lying in the intersection of the illuminated surface and the hyperboloid $H_{12}\left(s, s^{\prime}, t\right)=\left\{\boldsymbol{x}: r_{12}\left(s, s^{\prime}, \boldsymbol{x}\right)=c_{0} t\right\}$, which for flat topography, i.e. $\psi(\boldsymbol{x})=0$, is simply a hyperbola on the plane $x_{3}=0$. Thus an approximate inversion of $\mathcal{F}$ can be computed by a suitable backprojection:

$$
\begin{array}{r}
\mathcal{K}\left[\mathcal{F}\left[R_{G}\right]\right](\boldsymbol{z})=\int \mathrm{e}^{\mathrm{i} 2 \pi \omega\left(t-r_{12}\left(s, s^{\prime}, \boldsymbol{z}\right) / c_{0}\right)} Q\left(\boldsymbol{z}, \omega, s, s^{\prime}\right) \\
\times \mathcal{F}\left[R_{G}\right]\left(s, s^{\prime}, t\right) d t d \omega d s d s^{\prime},
\end{array}
$$

where $\mathcal{K}$ will be referred to as the (FBP) imaging operator and $Q$ is the filter to be determined next.

Substituting (9) into (11), we approximate $R_{G}$ by

$$
\tilde{R}_{G}(\boldsymbol{z})=\mathcal{K} \mathcal{F}\left[R_{G}\right](\boldsymbol{z})=\int L(\boldsymbol{z}, \boldsymbol{x}) R_{G}(\boldsymbol{x}) d \boldsymbol{x},
$$

where

$$
\begin{aligned}
L(\boldsymbol{z}, \boldsymbol{x})= & \int \mathrm{e}^{\mathrm{i} 2 \pi \omega\left[r_{12}\left(s, s^{\prime}, \boldsymbol{x}\right)-r_{12}\left(s, s^{\prime}, \boldsymbol{z}\right)\right] / c_{0}} \\
& \times Q\left(\boldsymbol{z}, \omega, s, s^{\prime}\right) A_{12}\left(s, s^{\prime}, \omega, \boldsymbol{x}, \boldsymbol{x}\right) d \omega d s d s^{\prime},
\end{aligned}
$$

is the point spread function. We would like to make $L(\boldsymbol{z}, \boldsymbol{x})$ as close as possible to the Dirac delta function $\delta(\boldsymbol{x}-\boldsymbol{z})=$ $\int \exp (\mathrm{i} 2 \pi(\boldsymbol{x}-\boldsymbol{z}) \cdot \boldsymbol{\xi}) d \boldsymbol{\xi}$. In this regard, we write

$$
\left[r_{12}\left(s, s^{\prime}, \mathbf{x}\right)-r_{12}\left(s, s^{\prime}, \mathbf{z}\right)\right]=(\boldsymbol{x}-\boldsymbol{z}) \cdot \boldsymbol{\Xi}\left(s, s^{\prime}, \boldsymbol{x}, \boldsymbol{z}\right),
$$

and for fixed $s^{\prime}, \boldsymbol{x}$ and $\boldsymbol{z}$, make the change of variables

$$
(s, \omega) \rightarrow \boldsymbol{\xi}=\frac{\omega}{c_{0}} \boldsymbol{\Xi}\left(s, s^{\prime}, \boldsymbol{x}, \boldsymbol{z}\right),
$$

in the integral of (13) to obtain

$$
\begin{aligned}
L(\boldsymbol{z}, \boldsymbol{x})=\int \mathrm{e}^{\mathrm{i} 2 \pi(\boldsymbol{x}-\boldsymbol{z}) \cdot \boldsymbol{\xi}} Q\left(\boldsymbol{z}, \boldsymbol{\xi}, s^{\prime}\right) \\
\quad \times A_{12}\left(\boldsymbol{\xi}, s^{\prime}, \boldsymbol{x}, \boldsymbol{x}\right) \eta\left(\boldsymbol{x}, \boldsymbol{z}, \boldsymbol{\xi}, s^{\prime}\right) d \boldsymbol{\xi} d s^{\prime},
\end{aligned}
$$

where $Q\left(\boldsymbol{z}, \boldsymbol{\xi}, s^{\prime}\right)=Q\left(\boldsymbol{z}, s(\boldsymbol{\xi}), \omega(\boldsymbol{\xi}), s^{\prime}\right)$, and $\eta\left(\boldsymbol{x}, \boldsymbol{z}, \boldsymbol{\xi}, s^{\prime}\right)=$ $|\partial(s, \omega) / \partial \boldsymbol{\xi}|$, is the determinant of the Jacobian that comes from the change of variables (15).

Using the method of stationary phase, under assumption (10), the leading-order contribution to $\tilde{R}_{G}$ is

$$
\begin{array}{r}
\tilde{R}_{G}(\boldsymbol{z}) \approx \int_{\Omega_{\boldsymbol{z}, s^{\prime}}} \mathrm{e}^{\mathrm{i} 2 \pi(\boldsymbol{x}-\boldsymbol{z}) \cdot \boldsymbol{\xi}} Q\left(\boldsymbol{z}, \boldsymbol{\xi}, s^{\prime}\right) A_{12}\left(\boldsymbol{\xi}, s^{\prime}, \boldsymbol{z}, \boldsymbol{z}\right) \\
\times \eta\left(\boldsymbol{z}, \boldsymbol{z}, \boldsymbol{\xi}, s^{\prime}\right) R_{G}(\boldsymbol{x}) d \boldsymbol{x} d \boldsymbol{\xi} d s^{\prime},
\end{array}
$$


where

$$
\begin{array}{r}
\Omega_{\boldsymbol{z}, s^{\prime}}=\left\{\boldsymbol{\xi}=\omega / c_{0} \boldsymbol{\Xi}\left(s, s^{\prime}, \boldsymbol{z}, \boldsymbol{z}\right) \mid A_{12}\left(\omega, s, s^{\prime}, \omega, \boldsymbol{z}, \boldsymbol{z}\right) \neq 0\right. \\
\left.s \in\left[s_{a}, s_{b}\right]\right\}
\end{array}
$$

with

$$
\boldsymbol{\Xi}\left(s, s^{\prime}, \boldsymbol{z}, \boldsymbol{z}\right)=D \psi(\boldsymbol{z}) \cdot\left[\frac{\mathbf{z}-\gamma_{R_{1}}(s)}{\left|\mathbf{z}-\gamma_{R_{1}}(s)\right|}-\frac{\mathbf{z}-\gamma_{R_{2}}\left(s+s^{\prime}\right)}{\left|\mathbf{z}-\gamma_{R_{2}}\left(s+s^{\prime}\right)\right|}\right],
$$

where

$$
D \phi(\boldsymbol{z})=\left[\begin{array}{ccc}
1 & 0 & \partial \psi(\boldsymbol{z}) / \partial z_{1} \\
0 & 1 & \partial \psi(\boldsymbol{z}) / \partial z_{2}
\end{array}\right]
$$

Here, we assume that the flight trajectories of the receivers are smooth and that the receiver antenna beam patterns are focused on one side of their flight trajectories so that the only illuminated critical point is $\boldsymbol{x}=\boldsymbol{z}$. Thus, with the choice of

$$
Q\left(\boldsymbol{z}, \omega, s, s^{\prime}\right)=\frac{\chi_{\Omega_{\boldsymbol{z}, s^{\prime}}}(\boldsymbol{\xi}(s, \omega))}{\eta\left(\boldsymbol{z}, \boldsymbol{z}, \boldsymbol{\xi}, s^{\prime}\right)} \frac{A_{12}^{*}\left(\omega, s, s^{\prime}, \boldsymbol{z}, \boldsymbol{z}\right)}{\left|A_{12}\left(\omega, s, s^{\prime}, \boldsymbol{z}, \boldsymbol{z}\right)\right|^{2}}
$$

where $\chi_{\Omega_{z, s^{\prime}}}$ is a smooth cut-off function equal to one in most of the interior of $\Omega_{\boldsymbol{z}, s^{\prime}}$ and zero in the exterior of $\Omega_{\boldsymbol{z}, s^{\prime}}$, (17) becomes

$$
\tilde{R}_{G_{c t}}(\boldsymbol{z}) \approx \int \mathrm{e}^{\mathrm{i}(\boldsymbol{x}-\boldsymbol{z}) \boldsymbol{\xi}} \chi_{\Omega_{\boldsymbol{z}, s^{\prime}}}(\boldsymbol{\xi}) R_{G}(\boldsymbol{x}) d \boldsymbol{x} d \boldsymbol{\xi} d s^{\prime} .
$$

Equation (22) shows that the image $\tilde{R}_{G_{c t}}$ is a band-limited version of $R_{G}$ whose frequency content, by (15), is determined by the union of $\Omega_{\boldsymbol{z}, s^{\prime}}$. Mircolocal analysis of (22) tells us that an edge at point $\boldsymbol{z}$ is visible if the direction $\boldsymbol{n}_{\boldsymbol{z}}$ normal to the edge is contained in the union $\cup_{s^{\prime}} \Omega_{z, s^{\prime}}[10,8,9]$. Thus by (22) one can only reconstruct the edges of $R_{G}$ that are visible.

\section{MF-FBP VERSUS C-FBP}

Given $d(s, t)$, in MF-FBP, we approximate the reflectivity function $G$ by [17]

$$
\tilde{G}(\boldsymbol{z}) \approx \int \mathrm{e}^{\mathrm{i} 2 \pi \omega\left(t-r_{1, \mathbf{y}}(s, \boldsymbol{z}) / c_{0}\right)} Q_{\mathbf{y}}^{M}(\omega, s, \boldsymbol{z}) d(s, t) d \omega d t d \boldsymbol{s}
$$

where the filter $Q^{M}$ is given by

$$
Q_{\mathbf{y}}^{M}(\omega, s, \boldsymbol{z})=\frac{\chi_{\Omega_{\boldsymbol{z}}^{M}}\left(\boldsymbol{\xi}^{M}(s, \omega)\right)}{\eta^{M}\left(\boldsymbol{z}, \boldsymbol{z}, \boldsymbol{\xi}_{1}\right)} \frac{A_{\mathbf{y}}^{M^{*}}(\omega, s, \boldsymbol{z})}{\left|A_{\mathbf{y}}^{M}(\omega, s, \boldsymbol{z})\right|^{2}} .
$$

Here

$\Omega_{\boldsymbol{z}}^{M}=\left\{\boldsymbol{\xi}^{M}=\left(\omega / c_{0}\right) \boldsymbol{\Xi}^{M}(s, \boldsymbol{z}) \mid A_{\mathbf{y}}^{M}(\omega, s, \boldsymbol{z}) \neq 0, s \in\left[s_{a}, s_{b}\right]\right\}$

with

$$
\boldsymbol{\Xi}^{M}(s, \boldsymbol{z})=D \psi(\boldsymbol{z}) \cdot\left[\frac{\mathbf{z}-\gamma_{R_{1}}(s)}{\left|\mathbf{z}-\gamma_{R_{1}}(s)\right|}+\frac{\mathbf{z}-\mathbf{y}}{|\mathbf{z}-\mathbf{y}|}\right],
$$

$\chi_{\Omega_{z}^{M}}$ is a smooth cut-off function equal to one in most of the interior of $\Omega_{\boldsymbol{z}}^{M}$ and zero in the exterior of $\Omega_{\boldsymbol{z}}^{M}$, and $\eta^{M}$ is the determinant of the Jacobian that comes from the change of variables [17]

$$
(s, \omega) \rightarrow \boldsymbol{\xi}^{M}=\omega / c_{0} \boldsymbol{\Xi}^{M}(s, \boldsymbol{z}) .
$$

Then, comparison of MF-FBP reconstruction formula (23) with C-FBP reconstruction formula (11) can be summarized as follows:
1. Correlation provides an extra degree of freedom, namely $s^{\prime}$;

2. MF-FBP reconstructs the mean of the target scene while CFBP reconstructs the variance;

3. MF-FBP backprojects the data onto the ellipsoids $E(s)=\{\boldsymbol{x}$ : $\left.r_{1, \mathbf{y}}(s, \boldsymbol{x})=c_{0} t\right\}$, which requires knowledge of the locations of both the transmitter and the receivers. On the other hand, C-FBP backprojects the correlation of the data onto the hyperboloids $\mathrm{H}_{12}$, which only requires the knowledge of receiver locations.

4. The frequency content of MF-FBP and C-FBP reconstructions are determined by the unions $\cup_{\left(s^{\prime}, \boldsymbol{z}\right)} \Omega_{\boldsymbol{z}, s^{\prime}}$ and $\cup_{\boldsymbol{z}} \Omega_{\boldsymbol{z}}^{M}$, respectively.

5. An edge at point $z$ is visible in MF-FBP and C-FBP reconstructions if the direction normal to the edge is contained in $\cup_{s^{\prime}} \Omega_{z, s^{\prime}}$ and $\Omega_{z}^{M}$, respectively.

\section{NUMERICAL SIMULATIONS}

In our numerical simulations, we considered a scene of size $[0,22] \times$ $[0,22] \mathrm{km}^{2}$ which is discretized by $128 \times 128$ pixels as illustrated in Figure 2(a); isotropic transmitter and receiver antennas; circular flight trajectories $\gamma_{R_{1}}(s)=\gamma_{R_{2}}(s)=\gamma(s)=(11+22 \cos s, 11+$ $22 \sin s, 6.5) \mathrm{km}$, uniformly sampled for $s \in[0,2 \pi)$ at 512 points; and a transmitter located at $(0,0,6.5) \mathrm{km}$ radiating a delta-like impulse. Thus we generate the projection data (see Figure 2(b)) using (1) for $A_{i, \mathbf{y}}(\omega, s, \boldsymbol{x})=(|\gamma(s)-\mathbf{x}||\mathbf{x}-\mathbf{y}|)^{-1}$.

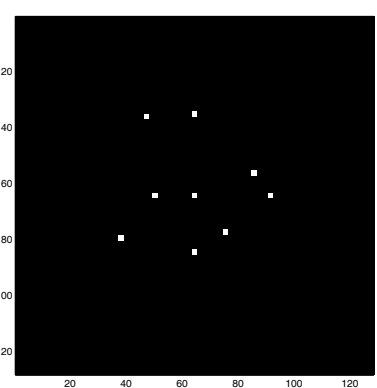

(a)

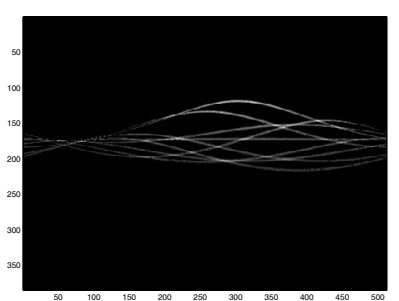

(b)
Fig. 2. (a) Scene used in numerical simulations. $(0,0,0) \mathrm{km}$ and $(22,22,0) \mathrm{km}$ are located at the upper left and lower right corners, respectively. (b) The projection data of the scene obtained using (1).

We implemented the inversion formulae (11) and (23). For fixed $s^{\prime},(11)$ requires to perform a FBP operation. Assuming that there are $\mathcal{O}(N)$ samples in both fast-time and slow-time variables, and the scene is sampled at $\mathcal{O}(N \times N)$ points, for each $s^{\prime}$ the FBP operation can be implemented in $\mathcal{O}\left(N^{3}\right)$ operations. Thus in total the presented inversion method requires $\mathcal{O}\left(N^{4}\right)$ operations. If fast backprojection methods are used $[7,14]$, the computational complexity of the reconstruction can be improved to $\mathcal{O}\left(N^{3} \log N\right)$. Similarly, (23) requires $\mathcal{O}\left(N^{3}\right)$ operations, which, using fast backprojection methods, can be improved up to $\mathcal{O}\left(N^{2} \log N\right)$.

The computational complexity of our implementation of the inversion formulae (11) and (23) are $\mathcal{O}\left(N^{4}\right)$ and $\mathcal{O}\left(N^{3}\right)$, respectively. The reconstructed images are presented in Figures 3(a) and 3(b), respectively.

Due to the choice of circular flight trajectories, all the edges of the scene are visible for both the C-FBP and the MF-FBP methods. Our numerical simulations demonstrate that the reconstructed image 


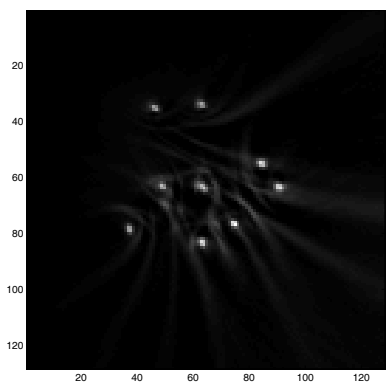

(a)

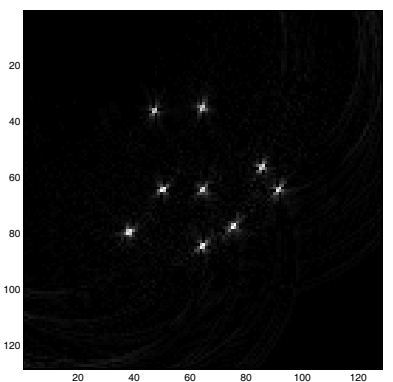

(b)
Fig. 3. Reconstructed images using (a) (11) and (b) (23).

using C-FBP is comparable to that of MF-FBP. Although C-FBP has a greater computational complexity than MF-FBP, C-FBP does not require the knowledge of the transmitter location, and can therefore be used for non-cooperative sources of opportunity. To demonstrate this, we replace the source-related terms in the reconstruction filter (21) by the function 1 and present the reconstructed image using the modified filter in Figure 4.

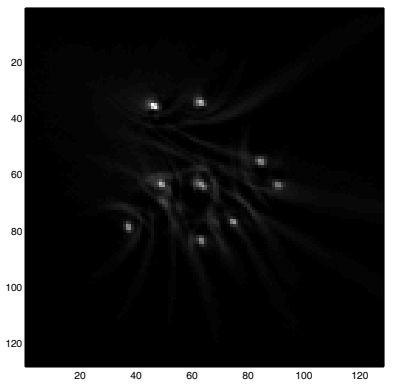

Fig. 4. Reconstructed image using (11) for a non-cooperative transmitter.

\section{CONCLUSION}

We presented a novel image reconstruction method for a bistatic synthetic-aperture hitchhiker system in the presence of a source of opportunity. We compared our method to the method introduced in [17] and demonstrated its performance in numerical simulations. Further properties and advantages of the presented method will be reported in our future research.

\section{REFERENCES}

[1] E.H Attia and B.D. Steinberg. Self-cohering large antenna arrays using the spatial correlation properties of radar clutter. IEEE Transactions on Antennas and Propagation, 37(1):3038, Jan. 1989.

[2] H.H. Barrett and K.J. Myers. Foundations of image science. Wiley-Interscience, Hoboken, N.J., 2004.

[3] T.-K. Chan, Y. Kuga, and A. Ishimaru. Experimental studies on circular SAR imaging in clutter using angular correlation function technique. IEEE Transactions on Geoscience and Remote Sensing, 37:2192-2197, Sep. 1999.
[4] M. Cherniakov, K. Kubik, and D. Nezlin. Radar sensors based on communication low earth orbiting satellites microwave emission. In Proceedings of IEEE International Geoscience and Remote Sensing Symposium, volume 3, pages 1007-1008, July 2000.

[5] M. Cherniakov, R. Saini, R. Zuo, and M. Antoniou. Space surface bistatic sar with space-borne non-cooperative transmitters. In Proceedings of European Radar Conference, pages 25-28, Oct. 2005.

[6] A.M. Horne and G. Yates. Bistatic synthetic aperture radar. In IEEE RADAR 2002, pages 6 - 10, Oct. 2002.

[7] S. Nilsson. Application of fast backprojection techniques for some inverse problems of integral geometry. $\mathrm{PhD}$ thesis, Linköping Studies in Science and Technology, 1997. Dissertation No. 499.

[8] C. J. Nolan and M. Cheney. Synthetic aperture inversion. Inverse Problems, 18:221-236, 2002.

[9] C.J. Nolan and M. Cheney. Synthetic aperture inversion for arbitrary flight paths and non-flat topography. IEEE Transactions on Image Processing, 12:1035-1043, 2003.

[10] E.T. Quinto. Singularities of the X-ray transform and limited data tomography in $r^{2}$ and $r^{3}$. SIAM J. Math. Anal., 24:12151225, 1993.

[11] Radar Systems Panel of the IEEE Aerospace and Electronic Systems Society. IEEE Standard Radar Definitions, 1997. IEEE Std 686-1997.

[12] M. Soumekh. Wide-bandwidth continuous-wave monostatic/bistatic synthetic aperture radar imaging. In Proceedings of International Conference on Image Processing, volume 3, pages 361-365, Oct. 1998.

[13] F. Treves. Introduction to Pseudodifferential and Fourier Integral Operators, volumes I and II. Plenum Press, NY, 1980.

[14] L.M.H. Ulander, H. Hellsten, and G. Stenström. Syntheticaperture radar processing using fast factorized back-projection. IEEE Transactions on Aerospace and electronic systems, 39:760-776, 2003.

[15] N.J. Willis. Bistatic radar. In M. Skolnik, editor, Radar Handbook, second edition, chapter 25. McGraw-Hill, New York, 1990.

[16] N.J. Willis. Bistatic Radar. Artech House, Norwood, MA, 1991.

[17] C.E. Yarman, B. Yazici, and M. Cheney. Bistatic synthetic aperture radar imaging for arbitrary flight trajectories and nonflat topography. submitted to IEEE Radar Conference, 2007. 